\title{
Câncer de mama: Análise da tendência de mortalidade em mulheres no Estado do
}

\section{Pará-Brasil}

\author{
Breast cancer: Analysis of the mortality trend in women in the State of Pará-Brazil \\ Cáncer de mama: Análisis de la tendencia de mortalidad en mujeres del Estado de Pará-Brasil
}

Recebido: 22/09/2021 | Revisado: 03/10/2021 | Aceito: 04/10/2021 | Publicado: 06/10/2021

\author{
Karolayne Assunção e Silva \\ ORCID: https://orcid.org/0000-0002-4071-4361 \\ Centro Universitário Metropolitano da Amazônia, Brasil \\ E-mail: karolayneassuncao99@gmail.com \\ Jorge Walber Pombo Marques Júnior \\ ORCID: https://orcid.org/0000-0003-2620-8267 \\ Centro Universitário Metropolitano da Amazônia, Brasil \\ E-mail: jorge_markes@hotmail.com \\ Larissa Pantoja Machado de Souza \\ ORCID: https://orcid.org/0000-0002-2700-3194 \\ Centro Universitário Metropolitano da Amazônia, Brasil \\ E-mail: larissapantoja@yahoo.com.br \\ Pedro Henrique Pereira Santos \\ ORCID: https://orcid.org/0000-0003-1238-2133 \\ Centro Universitário Metropolitano da Amazônia, Brasil \\ E-mail: pedrosantos91@ @otmail.com \\ Patrícia Danielle Lima de Lima \\ ORCID: https://orcid.org/0000-0002-1068-2813 \\ Universidade do Estado do Pará, Brasil \\ E-mail: patitalima@gmail.com \\ Sérgio Beltrão-Lima \\ ORCID: https://orcid.org/0000-0002-9531-2482 \\ Centro Universitário Metropolitano da Amazônia, Brasil \\ E-mail: sergio.lima@outlook.com \\ Symara Rodrigues-Antunes \\ ORCID: https://orcid.org/0000-0001-7346-0700 \\ Centro Universitário Metropolitano da Amazônia, Brasil \\ E-mail: symara@gmail.com \\ Danielle Cristinne Azevedo Feio \\ ORCID: https://orcid.org/0000-0002-0490-3704 \\ Centro Universitário Metropolitano da Amazônia, Brasil \\ E-mail: daniellefeio@yahoo.com.br
}

\begin{abstract}
Resumo
Introdução: $\mathrm{O}$ câncer de mama é o segundo tipo de neoplasia mais prevalente em mulheres, com aumento do número de óbitos a cada ano, apesar de ter elevada taxa de cura quando diagnosticado e tratado precocemente. Para tentar diminuir a taxa de mortalidade o Ministério da Saúde lançou a portaria n ${ }^{\circ}$ 189/2014 que visa instituir os Serviços de Referência para Diagnóstico de Câncer de Mama, contando com profissionais especializados e serviços diagnósticos. No entanto, há dificuldade de oferta de todos os procedimentos, impactando no diagnóstico definitivo e consequente aumento da mortalidade. Objetivo: Avaliar a tendência de mortalidade de câncer de mama no Pará, entre os anos de 2010 e 2018, a partir dos dados do Instituto Nacional do Câncer (INCA). Metodologia: Trata-se de um estudo observacional, descritivo, retrospectivo e comparativo baseado nos dados disponíveis no INCA. Resultados: No período de 2010 a 2018, no Pará houve 17,8 óbitos/100 mil habitantes por câncer de mama, abaixo dos valores observados comparativamente no Brasil $(27,7)$ e na região Norte $(19,1)$. No entanto, em Belém, a mortalidade foi maior, com média de 30 óbitos/100 mil habitantes. No comparativo entre as regiões do Brasil, o coeficiente de mortalidade da região Norte está acima das demais, tendo estas as seguintes médias: Sudeste 17,62; Nordeste 11,16; Sul 17,62 e Centro Oeste 12,68. Conclusão: A taxa de mortalidade por câncer de mama da Região Norte está acima das taxas das demais regiões do Brasil, tendo destaque para a cidade de Belém-Pará, onde a média de mortalidade está acima da registrada em todo o país.
\end{abstract}

Palavras-chave: Mortalidade; Epidemiologia; Estudos de séries temporais. 


\begin{abstract}
Introduction: Breast cancer is the second most prevalent type of cancer in women, with an increasing number of deaths each year, despite having a high cure rate when diagnosed and treated early. In order to try to reduce the mortality rate, the Ministry of Health launched ordinance ${ }^{\circ} 189 / 2014$ which aims to establish the Reference Services for Breast Cancer Diagnosis, with specialized professionals and diagnostic services. However, it is difficult to offer all procedures, impacting the definitive diagnosis and consequent increase in mortality. Objective: To assess the trend in breast cancer mortality in Pará, between 2010 and 2018, based on data from the National Cancer Institute (INCA). Methodology: This is an observational, descriptive, retrospective and comparative study based on data available at INCA. Results: In the period from 2010 to 2018, in Pará there were 17.8 deaths/100 thousand inhabitants due to breast cancer, below the values observed comparatively in Brazil (27.7) and in the North region (19.1). However, in Belém, mortality was higher, with an average of 30 deaths/100 thousand inhabitants. In the comparison between the regions of Brazil, the mortality coefficient in the North region is above the others, with the following averages: Southeast 17.62; Northeast 11.16; South 17.62 and Midwest 12.68. Conclusion: The mortality rate from breast cancer in the North Region is above the rates in other regions of Brazil, with emphasis on the city of Belém-Pará, where the average mortality rate is above that recorded throughout the country.
\end{abstract}

Keywords: Mortality; Epidemiology; Time series studies.

\title{
Resumen
}

Introducción: El cáncer de mama es el segundo tipo de cáncer más prevalente en mujeres, con un número creciente de muertes cada año, a pesar de tener una alta tasa de curación cuando se diagnostica y trata de manera temprana. En un intento por reducir la tasa de mortalidad, el Ministerio de Salud lanzó la ordenanza $n{ }^{\circ} 189 / 2014$, que tiene como objetivo establecer los Servicios de Referencia para el Diagnóstico del Cáncer de Mama, con profesionales especializados y servicios de diagnóstico. Sin embargo, es difícil ofrecer todos los procedimientos, lo que repercute en el diagnóstico definitivo y el consiguiente aumento de la mortalidad. Objetivo: Evaluar la tendencia de la mortalidad por cáncer de mama en Pará, entre 2010 y 2018, con base en datos del Instituto Nacional del Cáncer (INCA). Metodología: Se trata de un estudio observacional, descriptivo, retrospectivo y comparativo basado en datos disponibles en INCA. Resultados: En el período de 2010 a 2018, en Pará hubo 17,8 muertes/100 mil habitantes por cáncer de mama, por debajo de los valores observados comparativamente en Brasil $(27,7)$ y en la región Norte $(19,1)$. Sin embargo, en Belém, la mortalidad fue mayor, con un promedio de 30 muertes/100 mil habitantes. En la comparación entre las regiones de Brasil, el coeficiente de mortalidad en la región Norte está por encima de las demás, con los siguientes promedios: Sudeste 17,62; Noreste 11,16; Sur 17,62 y Medio Oeste 12,68. Conclusión: La tasa de mortalidad por cáncer de mama en la Región Norte está por encima de las tasas de otras regiones de Brasil, con énfasis en la ciudad de Belém-Pará, donde la tasa de mortalidad promedio es superior a la registrada en todo el país.

Palabras clave: Mortalidad; Epidemiologia; Estudios de series de tiempo.

\section{Introdução}

O câncer de mama é considerado um problema de saúde em nível mundial, sendo o tipo de neoplasia que mais acomete as mulheres, excluindo-se as lesões de pele "não melanomas". Além disso, é uma condição em que os óbitos vêm aumentando gradativamente nas cinco regiões territoriais do Brasil (Bezerra et al., 2018), possivelmente em função da elevada exposição a fatores de riscos, em especial nos grandes centros urbanos (Silva et al., 2017).

No estágio inicial, o câncer de mama é indolor e pode ser rastreado pelo autoexame, que deve ser feito preferencialmente sete dias após o início do ciclo menstrual. Nos casos de mulheres em menopausa deve-se escolher um dia por mês para realizá-lo, visto que as alterações provocadas pelo ciclo não estarão mais presentes (Batista et al., 2020; Müller et al., 2015).

No Brasil, os procedimentos para a confirmação diagnóstica do câncer de mama são realizados em serviços de saúde, como policlínicas ou hospitais de pequeno porte, caracterizados como atenção de média complexidade e como uma forma de facilitar o acesso a adesão da mulher ao atendimento em uma mesma unidade de saúde (Almeida et al., 2020). A fim de alcançar esse objetivo, o Ministério da Saúde lançou a Portaria $n^{\circ}$ 189/2014, instituindo os Serviços de Referência para Diagnóstico de Câncer de Mama (SDM), a partir de investimentos financeiros para sua posterior implantação.

A confirmação do diagnóstico é feita pela equipe médica com base no exame de mamografia. Tal exame é recomendado de ser feito como rastreamento de rotina em mulheres a partir dos 40 anos e em mulheres que possuam sinais e 
sintomas do câncer de mama. Além disso, em caso de presença de nodulações, a ultrassonografia acompanhada do exame histopatológico do nódulo também são exames utilizados para confirmação. Em caso de diagnóstico positivo para câncer de mama, inicia-se o tratamento imediatamente seguindo protocolos pré-estabelecidos que incluem, entre outros, radioterapia, quimioterapia e, em casos mais agressivos, a mastectomia. Esta consiste na retirada parcial ou total da mama através de procedimento cirúrgico (Brasil, 2014). Se o diagnóstico for precoce e o tratamento realizado de imediato, o câncer de mama apresenta taxas elevadas de sobrevida relativa (de Paiva \& Cesse, 2015).

Em 2014, segundo os dados do Sistema de Informações Ambulatoriais do Sistema Único de Saúde (SIA-SUS), apenas 46 serviços em todo o país informavam ter realizado todos os procedimentos necessários para a confirmação diagnóstica de mulheres com lesão/alteração descrita como suspeita de câncer de mama. A dificuldade de oferta de todos os procedimentos em um único serviço pode ter impacto direto na estratégia de diagnóstico precoce por gerar demanda por consultas intermediárias desnecessárias. No Brasil, entre 2010 e 2011, 40\% dos casos de câncer de mama chegaram aos hospitais de referência em oncologia sem o diagnóstico definitivo, o que indica insuficiência na estruturação da atenção secundária (INCA, 2016).

O tratamento é realizado, muitas vezes, de forma tardia, pois o tempo de espera para a realização de exames de diagnóstico é muito elevado. Aliado a esse aspecto há também a espera por leitos, que são poucos em comparação ao número de pacientes que necessitam de cirurgia, gerando consequências graves como a diminuição das chances de cura e do tempo de sobrevida do paciente (de Paiva \& Cesse, 2015; Teixeira \& Araújo, 2020; Leite et al., 2021).

O Ministério da Saúde determinou através da portaria No 876, de 16 de maio de 2013, que o tempo de espera entre o registro do diagnóstico e o início do tratamento não deve ultrapassar 60 dias (Brasil, 2013; Medeiros et al.,2015). No entanto, há outros aspectos que devem ser levados em consideração para o início do tratamento, principalmente nos estados das regiões Norte e Nordeste, como a distância domiciliar do paciente e o seu grau de instrução, que cursam negativamente nesse sentido (Santos et al., 2017).

O câncer de mama apresenta uma elevada taxa de mortalidade no mundo. No Brasil, e em especial nas regiões norte e nordeste, a demora ao acesso de pacientes às consultas especializadas dificultam um diagnóstico preciso e início precoce do seu tratamento, levando a um aumento cada vez mais significativo nas taxas de morbimortalidade. Diante do exposto, o presente estudo visa analisar os bancos de dados de mortalidade do câncer de mama em âmbito nacional, com ênfase no estado do Pará buscando avaliar a tendência de mortalidade desse CA, no período de 2010 e 2018, entre as faixas etárias.

\section{Metodologia}

Trata-se de estudo ecológico observacional de caráter descritivo, retrospectivo e comparativo de série histórica baseado em dados disponíveis na plataforma do Instituto Nacional do Câncer (INCA). O presente estudo foi desenvolvido em vinte meses, no período de março de 2019 a novembro de 2020. Para a coleta de dados foram incluídos os indivíduos do sexo feminino, a partir de 30 anos, faixa etária de maior incidência dos casos, portadoras de câncer de mama entre os anos de 2010 e 2018. Com base nesses critérios foram realizadas as filtragens nos dados estatísticos sobre o Brasil, a região Norte, o estado do Pará e a cidade de Belém.

Os dados foram analisados de acordo com as descrições, comparações e interpretações das informações expostas nos bancos de dados. A taxa de mortalidade por câncer de mama foi calculada por 100.000 habitantes e ajustada pelo método direto por faixas etárias em intervalos de 10 anos. A distribuição percentual da população brasileira disponibilizada pelo Instituto Brasileiro de Geografia e Estatística (IBGE), através do Censo de 2010, foi utilizada como padrão.

Em relação ao cálculo das taxas de variação percentual anual (VPA), utilizou-se a regressão de Prais-Winsten, com a correção de autocorrelação de primeira ordem. A variável independente foram os anos de 2010 a 2018, já em relação a variável 
dependente esta foi o logaritmo (na base 10). A partir da regressão obteve-se o valor estimado do coeficiente "b", o erro padrão da estimativa e o valor da estatística " $t$ " (da distribuição $t$ de Student), os quais foram usados para derivar a VPA e o respectivo intervalo de confiança. A tendência de declínio, estagnação ou aumento da tendência de mortalidade foi expressa como VPA, com intervalo de confiança de $95 \%$, e foi considerada como estacionária a tendência cujo intervalo de confiança não era diferente de zero.

Em relação a realização da análise estatística descritiva, a confecção das tabelas e gráficos, foi utilizado o software Microsoft Excel 2010. Para a análise de tendência das séries temporais e regressão de Prais-Winsten foram utilizados os programas Microsoft Excel e SPSS (versão 20.0, por meio do comando AREG). A avaliação por um Comitê de Ética em Pesquisa não se fez necessário por se tratar de dados do tipo secundários e de domínio público, provenientes da base de dados públicos do INCA.

\section{Resultados}

Segundo o banco de dados do INCA, no período de 2010 a 2018, houveram 133 mil casos de câncer de mama em mulheres com 30 anos ou mais no país, o que corresponde a $16 \%$ de todos os casos de neoplasia ocorridos no período, incluindo os de pele não melanoma, cujo número total de casos foi de 822.608 em mulheres com 30 anos ou mais. A região Norte respondeu por 5.123 casos de câncer de mama, o que correspondeu a 3,8\% de todos os casos de câncer de mama do Brasil no período, sendo que 2.292 casos (45\%) ocorreram no Pará. Na cidade de Belém ocorreram quase metade dos casos (1.025) do Estado do Pará (Tabela 1). No Brasil, houve coeficiente médio de cerca de 27,7 óbitos por câncer de mama por ano, a cada 100 mil habitantes. As taxas na região Norte como um todo e no Pará ficaram abaixo da taxa do país. No entanto, em Belém, o valor associado a taxa de mortalidade foi maior que a nacional, com coeficiente médio de 30,4 óbitos a cada 100 mil habitantes.

Tabela 1 - Óbitos por câncer de mama, número total e porcentagem, coeficiente médio por 100.000 habitantes e tendência das taxas de mortalidade em mulheres a partir de 30 anos de idade, segundo o local de 2010-2018.

\begin{tabular}{|c|c|c|c|c|c|c|}
\hline \multirow[t]{2}{*}{ Local } & \multicolumn{2}{|c|}{ Óbitos ${ }^{1}$} & \multirow{2}{*}{$\begin{array}{c}\text { Coef. } \\
\text { Médio }^{2}\end{array}$} & \multirow[t]{2}{*}{$\mathbf{V P A}^{3}$} & \multirow[t]{2}{*}{ IC-95\% ${ }^{4}$} & \multirow[t]{2}{*}{ Interpretação } \\
\hline & $\mathbf{N}$ & $\%$ & & & & \\
\hline Brasil & 133.050 & 16,17 & 27,7 & 2,3 & 0,01 a 4,70 & Aumento \\
\hline Norte & 5.123 & 13,24 & 19,1 & 5,7 & 5,20 a 6,16 & Aumento \\
\hline Pará & 2.292 & 13,43 & 17,8 & 5,9 & 5,02 a 6,84 & Aumento \\
\hline Belém & 1.025 & 16,10 & 30,4 & 4,5 & $-0,11$ a 9,26 & Aumento \\
\hline
\end{tabular}

${ }^{1}$ : As percentagens de óbitos são proporcionais ao total de óbitos por todas as neoplasias no período.

2: Número médio anual de óbitos por 100.000 habitantes, padronizado por faixas etárias da população brasileira, IBGE/Censo-2010.

3: Variação Percentual Anual.

${ }^{4}$ : Intervalo de confiança do VPA.

Fonte: Autores.

Observou-se tendência de aumento percentual médio da taxa de mortalidade no Pará e na região Norte no período estudado. No entanto, a VPA no Pará foi maior que no Norte (variação percentual média de 5,9 e 5,7 respectivamente, além de ser maior que a VPA estável observada no país. Em Belém, também houve uma tendência de aumento da taxa de mortalidade (IC95\% de -0,11 a 9,26\%), em um patamar mais elevado que em comparação à variação média nacional (Tabela 1). 
A região Norte apresentou 5.198 óbitos por câncer de mama no período entre 2010 e 2018, com a taxa bruta de 19,1 óbitos para cada 100.000 habitantes em indivíduos do sexo feminino. A fim de comparar os dados colhidos no Norte com as diferentes regiões do Brasil, verificamos que a Região Sudeste obteve 68.061 óbitos por câncer de mama notificados com taxa bruta de 17,62; na região Nordeste houve 28.508 óbitos, sendo a taxa bruta 11,16; na região Sul ocorreram 23.738 óbitos com 17,62 de taxa bruta; e por último a região Centro-oeste registrou 8.612 óbitos apresentando 12,68 da referida taxa por cem mil habitantes.

Ao comparar as regionais de saúde do Estado do Pará, observou-se que a maior taxa de mortalidade foi na a Região Metropolitana que compreende os municípios de Belém, Ananindeua, Santa Bárbara, Benevides e Marituba com taxa de 13,29 por 100.000 habitantes; a região que apresentou menor taxa de mortalidade foi a região Araguaia-Xingu com 1,43 por 100.000 habitantes, compreendendo os municípios de Altamira, Anapú, Brasil Novo, Medicilândia, Pacajá, Placas, Porto de Moz, Senador José Porfírio, Uruará, Vitória do Xingu, Agua Azul do Norte, Bannach, Conceição do Araguaia, Cumaru do Norte, Floresta do Araguaia, Ourilândia do Norte, Pau D'arco, Redenção, Rio Maria, Santa Maria das Barreiras, Santana do Araguaia, São Felix do Xingu, Sapucaia, Tucumã e Xinguara.

A Figura 1 demonstra a série histórica das taxas de mortalidade por câncer de mama na cidade de Belém, no Estado do Pará, região Norte e no Brasil. Observa-se a tendência de aumento da taxa nacional (em azul), porém aumento mais acentuado no Norte e no Pará, comparativamente. Observa-se, também, que a taxa de óbitos em Belém por câncer de mama tem se mantido acima da nacional, com exceção dos anos de 2010 e 2012, e que a taxa de óbitos por câncer de mama no Pará segue tendência similar à da região Norte.

Figura 1 - Tendência das taxas de mortalidade por câncer de mama, ajustadas por faixa etária, no sexo feminino, Belém, Pará, Norte e Brasil, 2010-2018.

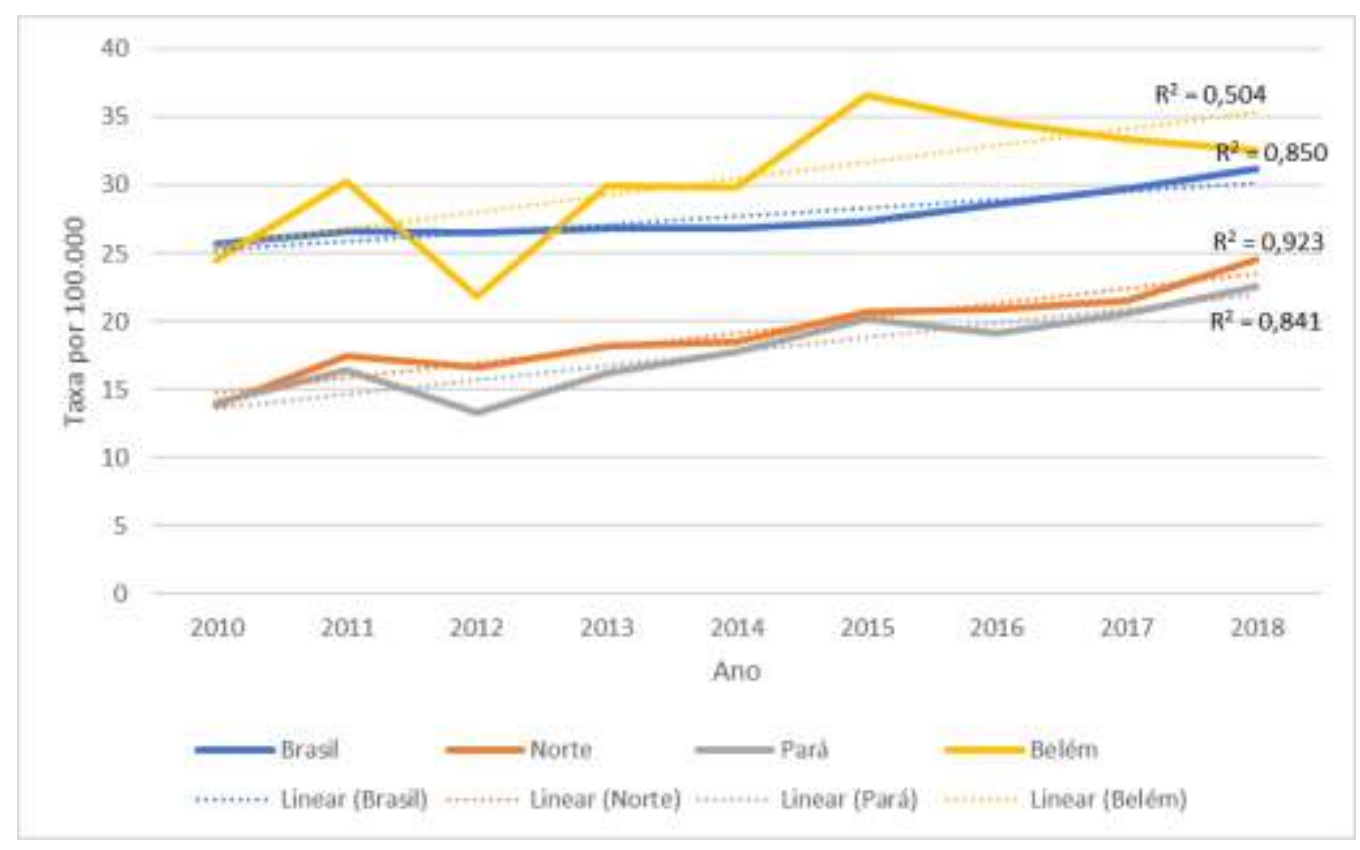

Fonte: Autores.

A distribuição das taxas de mortalidade por faixa etária e local é demonstrada na Tabela 2. Observa-se que a mortalidade aumenta conforme a idade, chegando a 109 óbitos por 100 mil habitantes no país em mulheres com 80 anos ou mais. Em Belém, essa taxa é superior a nacional, com 113 óbitos por 100 mil habitantes no período considerado, e cerca de 73\% maior que a mortalidade observada no Norte ou no Pará. Entretanto, foi observado na região Norte uma diferença 
significativa do ponto de vista quantitativo, entre as faixas de idades, sendo a faixa entre 40 a 49 anos com 1.205 óbitos e em seguida dos pacientes entre 50 a 59 anos apresentando 1.363 óbitos, em relação às demais faixas etárias (p=0.0019).

Tabela 2 - Taxa de mortalidade ajustada por idade, para cada 100.000 habitantes, por câncer de mama no sexo feminino, Belém, Pará, Norte e Brasil, 2010-2018.

\begin{tabular}{lcccccc}
\hline \multicolumn{1}{c}{ Local } & \multicolumn{6}{c}{ Faixa Etária } \\
\cline { 2 - 7 } & 30 a 39 & 40 a 49 & 50 a 59 & 60 a 69 & 70 a 79 & 80 OU + \\
\hline Brasil & 5,97 & 18,29 & 33,62 & 47,49 & 63,96 & 109,24 \\
Norte & 4,40 & 14,60 & 24,73 & 31,45 & 40,78 & 65,31 \\
Pará & 4,23 & 13,56 & 22,11 & 28,09 & 39,22 & 65,55 \\
Belém & 5,90 & 22,68 & 35,90 & 51,89 & 71,88 & 113,37 \\
\hline
\end{tabular}

Fonte: Autores.

\section{Discussão}

Os resultados analisados nesta pesquisa apontaram que, do total de casos da Região Norte no período estudado, o Estado do Pará é responsável por 45\% dos casos de câncer de mama e desses, metade está associado à sua capital, Belém. Tais observações podem estar diretamente ligadas à quantidade de mamógrafos na região Norte que é 1 para cada 100.000 habitantes. Esta quantidade acaba por inviabilizar o diagnóstico precoce, especialmente nas áreas de mais difícil acesso. É de conhecimento que o diagnóstico e tratamento precoce aumentam as chances de cura da paciente. Contudo, o que se observa no Pará e na região Norte como um todo é que muitas acabam por serem diagnosticadas em fase avançada do desenvolvimento da neoplasia, o que atrasa o tratamento e impacta diretamente no aumento da taxa de mortalidade (Tomazelli et al., 2017).

Todavia, em países em desenvolvimento como o Brasil, as taxas de mortalidade são superiores em relação a países desenvolvidos, pois mesmo com os investimentos governamentais recentes na implantação do SDM, a dificuldade ao acesso e até mesmo a presença de áreas descobertas pelo serviço, tornam o diagnóstico mais tardio o que, associado ao elevado custo do tratamento e a burocracia do sistema de saúde pública, acabam por tornar-se os principais entraves para a cura da doença (de Paiva \& Cesse, 2015).

Além disso, os dados também revelam que há uma ineficiência da atenção básica na região Norte do país com relação ao câncer de mama. Em muitas regiões isoladas existe a inoperância parcial e/ou total do serviço da saúde, além de muitas cidades possuírem uma equipe de saúde muito pequena ou única para um contingente populacional vasto, o que pode levar a demora para conseguir ter acesso ao atendimento, com constante encaminhamento para as capitais, especificamente a capital Belém, que possui centros de especialidades que podem atender ou diagnosticar. Contudo, geralmente nessas capitais há uma fila de espera grande, atrasando ainda mais o diagnóstico e tratamento da neoplasia mamária (Kluthcovsky et al.,2014; Guerra et al., 2020).

A concentração de metade do número de óbitos por câncer de mama na capital Belém pode ser consequência direta de uma possível falha nos registros de câncer existentes no país, como por exemplo, na notificação errônea associada ao local de morte do paciente (Guerra et al., 2005). Como já exposto, os pacientes que não conseguem diagnóstico e/ou tratamento em seu domicílio de residência, são encaminhados à capital, devido as grandes distâncias de alguns municípios para Belém e a necessidade de acesso aos serviços de saúde, alguns pacientes podem tem que se mudar, mesmo que temporariamente (Saldanha et al., 2009). Quando esta paciente eventualmente vai a óbito, ele pode erroneamente ser associado ao local de 
ocorrência dele e não ao local de origem da paciente, que somente se deslocou para realizar tratamento, uma vez que estudos anteriores de outras patologias demostraram fragilidades do sistema de vigilância epidemiológica associadas ao preenchimento irregular dos registros por motivos variados, e até mesmo problemas estruturais, como fatos que comprometem a qualidade da informação (Barbosa et al., 2015; Pedroso et al., 2020). Outros fatores também estão presentes como a falta de cultura de rastreio de doenças quando aparecem sinais e sintomas; a não realização do autoexame das mamas e não cumprimento dos protocolos de diagnóstico semelhante ao que acontece em outras regiões do país (Borges et al., 2016).

A indisponibilidade em muitas regiões de especialistas na área e equipamentos como mamógrafos para diagnóstico, são fatores que também podem estar associados a essas altas taxas de mortalidade, uma vez que causa impacto direto no diagnóstico e tratamento dos pacientes (Xavier et al., 2016; Silva et al., 2018). Na série histórica analisada neste estudo observou-se uma tendência de aumento percentual médio da taxa de mortalidade global concordante com diversas pesquisas (Kluthcovsky et al.,2014; Couto et al., 2018; de Oliveira Barros et al., 2020).

Os fatos observados são corroborados, por uma pesquisa realizada no estado do Tocantins, o qual demonstra que em até $51,1 \%$ dos casos encontrados a doença já está em estágio e fase avançada dificultando assim o tratamento. A maioria de mulheres diagnosticadas no Estado do Tocantins são provenientes do sudeste do Pará, o que evidencia que as mulheres são encaminhadas para Estados adjacentes para tentar realizar o diagnóstico e tratamento devido à grande fila de espera na cidade de Belém (Suleiman et al., 2017).

Em outras regiões do país, as taxas de mortalidade se mantiveram estáveis e, em algumas, até decresceram (como na região sul). Isso pode ser resultado da maior eficiência da atenção básica que consegue diagnosticar precocemente e encaminhar para uma rede de atenção secundária e terciária que conseguem manejar a paciente com maior oferta de exames e tratamentos, impactando na diminuição da taxa de mortalidade por neoplasia mamária (Silva \& Riul, 2011; Rodrigues et al., 2015; Prolla et al., 2015). Portanto, isso evidencia a importância e a necessidade urgente de políticas públicas em saúde mais efetivas voltadas a região Norte.

De acordo com os dados apresentados, há pouca diferença nos índices de mortalidade nas faixas etárias de 30 a 39 e 40 a 49 anos em Belém, no Pará, na região Norte e no Brasil. Já na faixa etária a partir de 60 anos, a taxa de mortalidade aumenta de maneira exacerbada em todas as regiões apresentadas observando-se uma discrepância na cidade de Belém em relação ao Pará. Em Belém o índice de mortalidade por câncer de mama está acima desse padrão do Estado do Pará, cidade essa que se destaca por ser o centro de referência para o diagnóstico e para tratamento, tendo assim um número elevado de pacientes encaminhadas de outras cidades. Portanto, o estudo demonstra a necessidade de políticas públicas visando a descentralização do tratamento e do diagnóstico e consequentemente a prevenção em regiões mais remotas do estado do Pará.

\section{Conclusão}

As taxas de mortalidade por 100 mil habitantes pelo câncer de mama em mulheres na região Norte, Pará e Belém são maiores do que comparados com outras regiões do país, demonstrando possíveis problemas de atenção primária, logística de saúde e falha no diagnóstico precoce, associados também a possíveis altos índices de subnotificação de novos casos se tornando um grave problema de saúde pública. Dessa forma, são necessárias mais pesquisas que avaliem a cobertura dos serviços em saúde do Estado do Pará e dentro da cidade de Belém, para que assim haja o desenvolvimento de políticas públicas direcionadas a construção e ampliação de redes de saúde integradas entre as atenções primária, secundária e terciária.

\section{Referências}

Almeida, M. M. M., Almeida, P. F. D., \& Melo, E. A. (2020). Regulação assistencial ou cada um por si? Lições a partir da detecção precoce do câncer de mama em redes regionalizadas do Sistema Único de Saúde (SUS). Interface-Comunicação, Saúde, Educação, 24. https://doi.org/10.1590/Interface.190609 
Barbosa, J. R., Barrado, J. C. D. S., Zara, A. L. D. S. A., \& Siqueira Júnior, J. B. (2015). Avaliação da qualidade dos dados, valor preditivo positivo, oportunidade e representatividade do sistema de vigilância epidemiológica da dengue no Brasil, 2005 a 2009. Epidemiologia e Serviços de Saúde, 24, 49-58. https://doi.org/10.5123/S1679-49742015000100006

Batista, G. V., Moreira, J. A., Leite, A. L., \& Moreira, C. I. H. (2020). Câncer de mama: fatores de risco e métodos de prevenção. Research, Society and Development, 9(12), e15191211077-e15191211077. https://doi.org/10.33448/rsd-v9i12.11077

Bezerra, H. D. S., Melo, T. F. V. D., Barbosa, J. V., Feitosa, E. E. L. C., \& Sousa, L. C. M. (2018). Avaliação do acesso em mamografias no Brasil e indicadores socioeconômicos: um estudo espacial. Revista Gaúcha de Enfermagem, 39. e20180014. https://doi.org/10.1590/1983-1447.2018.20180014

Borges, Z. D. S., Wehrmeister, F. C., Gomes, A. P., \& Gonçalves, H. (2016). Exame clínico das mamas e mamografia: desigualdades nas regiões Sul e Nordeste do Brasil. Revista Brasileira de Epidemiologia, 19, 1-13. https://doi.org/10.1590/1980-5497201600010001

Brasil, Ministério da Saúde, Portaria n ${ }^{\circ}$ 189, de 31 de janeiro de 2014. Institui o Serviço de Referência para Diagnóstico e Tratamento de Lesões Precursoras do Câncer do Colo de Útero (SRC), o Serviço de Referência para Diagnóstico de Câncer de Mama (SDM) e os respectivos incentivos financeiros de custeio e de investimento para a sua implantação.

Brasil, Ministério da saúde, Portaria N $\mathrm{N}^{\mathrm{o}}$ 876, de 16 de maio de 2013, Dispõe sobre a aplicação da Lei $\mathrm{n}^{\mathrm{o}} 12.732$, de 22 de nove mbro de 2012 , que versa a respeito do primeiro tratamento do paciente com neoplasia maligna comprovada, no âmbito do Sistema Único de Saúde (SUS).

Couto, M. S. D. A., Guerra, M. R., Firme, V. D. A. C., \& Bustamante-Teixeira, M. T. (2018). Comportamento da mortalidade por câncer de mama nos municípios brasileiros e fatores associados. Revista Panamericana de Salud Pública, 41, e168. https://doi.org/10.26633/RPSP.2017.168

de Oliveira Barros, L., Menezes, V. B. B., Jorge, A. C., de Morais, S. S. F., \& da Silva, M. G. C. (2020). Mortalidade por Câncer de Mama: uma Análise da Tendência no Ceará, Nordeste e Brasil de 2005 a 2015. Revista Brasileira de Cancerologia, 66(1). https://doi.org/10.32635/2176-9745.RBC.2020v66n1.740

de Paiva, C. J. K., \& Cesse, E. Â. P. (2015). Aspectos relacionados ao atraso no diagnóstico e tratamento do câncer de mama em uma Unidade Hospitalar de Pernambuco. Revista Brasileira de Cancerologia, 61(1), 23-30. https://doi.org/10.32635/2176-9745.RBC.2015v61n1.374

Guerra, M. R., de Moura Gallo, C. V., Azevedo, G., \& Mendonça, S. (2005). Risco de câncer no Brasil: tendências e estudos epidemiológicos mais recentes. Revista brasileira de cancerologia, 51(3), 227-234. https://doi.org/10.32635/2176-9745.RBC.2005v51n3.1950

Guerra, M. R., Nogueira, M. C., Malta, D. C., Côrrea, C. S. L., de Souza, M. D. F. M., Curado, M. P., \& Bustamante-Teixeira, M. T. (2020). Inequalities in the burden of female breast cancer in Brazil, 1990-2017. Population health metrics, 18(1), 1-13. https://doi.org/10.1186/s12963-020-00212-5

Instituto Nacional de Câncer José Alencar Gomes da Silva. (2016) Informativo detecção precoce. Ano 6, http://www1.inca.gov.br/inca/Arquivos/informativo_numero3_2015.versao_2016.pdf

Kluthcovsky, A. C. G. C., Faria, T. N. P., Carneiro, F. H., \& Strona, R. (2014). Female breast cancer mortality in Brazil and its regions. Revista da Associação Médica Brasileira, 60, 387-393. https://doi.org/10.1590/1806-9282.60.04.019

Leite, C. G., Ruhnke, B. F., \& Valejo, F. A. M. (2021). Correlação entre tempo de diagnóstico, tratamento e sobrevida em pacientes com câncer de mama: uma revisão de literatura. Colloquium Vitae. ISSN: 1984-6436, 13(1), 12-16. https://doi.org/10.5747/cv.2020.v13.n1.v318

Medeiros, G. C., Bergmann, A., Aguiar, S. S. D., \& Thuler, L. C. S. (2015). Análise dos determinantes que influenciam o tempo para o início do tratamento de mulheres com câncer de mama no Brasil. Cadernos de Saúde Pública, 31, 1269-1282. https://doi.org/10.1590/0102-311X00048514

Müller, M. C., Frasson, A., Kieling, C., Hoffmann, F. S., Fleck, P., Zogbi, H., ... \& Werres, J. (2005). A prática do auto-exame das mamas em mulheres de uma comunidade universitária. Psico-USF, 10, 185-190. https://doi.org/10.1590/S1413-82712005000200010

Pedroso, A. O., Nogueira, L. M. V., Rodrigues, I. L. A., Trindade, L. D. N. M., \& D'Annibale, V. L. A. (2020). Análise do sistema de vigilância epidemiológica da febre de chikungunya no estado do Pará. Cogitare Enfermagem, 25. http://dx.doi.org/10.5380/ce.v25i0.65540

Prolla, C. M. D., Silva, P. S. D., Netto, C. B. O., Goldim, J. R., \& Ashton-Prolla, P. (2015). Knowledge about breast cancer and hereditary breast cancer among nurses in a public hospital. Revista latino-americana de enfermagem, 23(1), 90-97. https://doi.org/10.1590/0104-1169.0185.2529

Rodrigues, J. D., Cruz, M. S., \& Paixão, A. N. (2015). Uma análise da prevenção do câncer de mama no Brasil. Ciência \& saúde coletiva, 20, $3163-3176$. https://doi.org/10.1590/1413-812320152010.20822014

Saldanha, R. D. F., Xavier, D. R., Carnavalli, K. D. M., Lerner, K., \& Barcellos, C. (2019). Estudo de análise de rede do fluxo de pacientes de câncer de mama no Brasil entre 2014 e 2016. Cadernos de Saúde Pública, 35. https://doi.org/10.1590/0102-311X00090918

Santos, A. E. S., Araújo, T. M., Pimentel, M. S. L., de Andrade, J. S., \& Gonçalves, L. L. C. (2017, December). Fatores Relacionados a não Adesão ao Rastreamento para o Câncer de Mama no Brasil: uma Revisão Integrativa. In Congresso Internacional de Enfermagem (Vol. 1, No. 1). Retrieved May 30, 2020, from https://eventos.set.edu.br/cie/article/view/5523

Silva, G. A., Souza-Júnior, P. R. B. D., Damacena, G. N., \& Szwarcwald, C. L. (2017). Detecção precoce do câncer de mama no Brasil: dados da Pesquisa Nacional de Saúde, 2013. Revista de Saúde Pública, 51, 14s. https://doi.org/10.1590/S1518-8787.2017051000191

Silva, M. T. A. D., Silva, V. B. D., Mangueira, J. D. O., Gurgel, G. D., \& Leal, E. M. M. (2018). Distribuição dos mamógrafos e da oferta de mamografia em relação ao parâmetro assistencial do Sistema Único de Saúde em Pernambuco. Revista Brasileira de Saúde Materno Infantil, 18, 609-618. https://doi.org/10.1590/1806-93042018000300009

Silva, P. A. D., \& Riul, S. D. S. (2011). Câncer de mama: fatores de risco e detecção precoce. Revista Brasileira de Enfermagem, 64, 1016-1021. https://doi.org/10.1590/S0034-71672011000600005 
Research, Society and Development, v. 10, n. 13, e109101320929, 2021

(CC BY 4.0) | ISSN 2525-3409 | DOI: http://dx.doi.org/10.33448/rsd-v10i13.20929

Suleiman, N. N., Nascimento, N., Botelho, J. M. S., \& Coelho, R. C. (2017). Panorama do câncer de mama em mulheres no norte do Tocantins-Brasil. Revista do Colégio Brasileiro de Cirurgiões, 44, 316-322. https://doi.org/10.1590/0100-69912017004001

Teixeira, L. A., \& Araújo, L. A. (2020). Câncer de mama no Brasil: medicina e saúde pública no século XX. Saúde e Sociedade, 29. https://doi.org/10.1590/S0104-12902020180753

Tomazelli, J. G., \& Silva, G. A. (2017). Rastreamento do câncer de mama no Brasil: uma avaliação da oferta e utilização da rede assistencial do Sistema Único de Saúde no período 2010-2012. Epidemiologia e Serviços de Saúde, 26, 713-724. https://doi.org/10.5123/S1679-49742017000400004

Xavier, D. R., Oliveira, R. A. D. D., Matos, V. P. D., Viacava, F., \& Carvalho, C. D. C. (2016). Cobertura de mamografias, alocação e uso de equipamentos nas Regiões de Saúde. Saúde em debate, 40, 20-35. https://doi.org/10.1590/0103-1104201611002 